\title{
Lidil
}

Revue de linguistique et de didactique des langues

36 | 2007

Échanges exolingues via internet et appropriation des langues-cultures

\section{Échanges exolingues et interculturalité dans un environnement informatisé plurilingue}

Christian Degache, Covadonga Lòpez Alonso et Arlette Séré

\section{(2) OpenEdition}

Journals

Édition électronique

URL : http://journals.openedition.org/lidil/2473

DOI : $10.4000 /$ lidil. 2473

ISSN : 1960-6052

Éditeur

UGA Éditions/Université Grenoble Alpes

Édition imprimée

Date de publication : 1 décembre 2007

Pagination : $93-118$

ISBN : 978-2-84310-108-3

ISSN : $1146-6480$

Référence électronique

Christian Degache, Covadonga Lòpez Alonso et Arlette Séré, «Échanges exolingues et interculturalité dans un environnement informatisé plurilingue », Lidil [En ligne], 36 | 2007, mis en ligne le 03 juin 2009, consulté le 10 décembre 2020. URL : http://journals.openedition.org/lidil/2473 ; DOI : https://doi.org/ 10.4000/lidil.2473

Ce document a été généré automatiquement le 10 décembre 2020.

(C) Lidil 


\title{
Échanges exolingues et interculturalité dans un
} environnement informatisé
plurilingue

\author{
Christian Degache, Covadonga Lòpez Alonso et Arlette Séré
}

1 Dans un environnement de formation plurilingue, on peut considérer, compte tenu de la conscience des participants d'être dans un espace réunissant des individus dotés de compétences linguistiques globalement asymétriques, que la majorité des échanges est de nature exolingue. Ainsi en est-il sur la plateforme Galanet dédiée à l'intercompréhension en langues romanes. Certes les échanges endolingues sont toujours possibles entre locuteurs d'une même langue mais cela n'empêche que l'exolinguisme est omniprésent à travers différents types d'échanges exolingues - unilingues, bilingues, plurilingues - en fonction des compétences linguistiques des interactants et des langues réellement impliquées dans l'échange (Degache, 2006b).

2 Dès lors, il y a lieu de se demander si toutes ces interactions sont également de nature interculturelle. Bien des éléments peuvent plaider en faveur de cette position: les attentes et motivations des participants, les bilans effectués en fin de formation, les thématiques abordées. Pourtant, nous savons qu'il ne suffit pas de parler d'interculturel pour que la dimension interculturelle soit réellement en jeu dans l'échange. Nous reprenons ici les positions de Dausendschön-Gay et Kraft (1998 : 95-96), qui, d'un point de vue " délibérément ethnométhodologique » considèrent « qu'il ne faut pas confondre les données préalables à l'interaction - en particulier le savoir concernant l'appartenance culturelle et linguistique des interactants - et la situation que ces interactants construisent tout au long de l'interaction ». La question centrale sera donc ici la suivante : dans le cadre des sessions d'intercompréhension plurilingue sur la plateforme Galanet, à quels moments du scénario, sous quelles formes et avec quelles caractéristiques et finalités, la dimension interculturelle est-elle mobilisée dans l'interaction? 
Dans cet article, nous commencerons par situer l'interculturalité dans les orientations actuelles de la didactique des langues, en précisant la place qui lui est accordée dans la perspective actionnelle prônée par le Cadre européen de référence et le cadre théorique de notre réflexion, inspirée de travaux sur la communication exolingue conduits selon une approche ethnométhodologique. Ce afin de pouvoir mener à bien une catégorisation des manifestations interculturelles dans l'interaction plurilingue en prenant appui sur un certain nombre de données tirées de l'environnement de formation en question.

\section{Interculturel, didactique des langues et analyse conversationnelle}

\section{Orientations actuelles}

4 Le Cadre européen commun de référence pour les langues (CECR) concrétise les travaux menés depuis plus d'une vingtaine d'années autour de la compétence interculturelle en didactique des langues (voir les références données par Mangenot et Zourou ici même). Elle y apparait (Conseil de l'Europe, $2001:$ 83) dans les compétences générales du sujet en tant que savoir dynamique issu d'une prise de conscience contrastive entre culture d'origine et culture cible, située dans " un plus grand éventail de cultures » et tenant compte «de la manière dont chaque communauté apparait dans l'optique de l'autre, souvent sous forme de stéréotypes nationaux ». Cette conception permet de délimiter quatre savoir-faire (ibid. : 84) :

1. la capacité d'établir une relation entre la culture d'origine et la culture étrangère ;

2. la sensibilisation à la notion de culture et la capacité de reconnaitre et d'utiliser des stratégies variées pour établir le contact avec des gens d'une autre culture ;

3. la capacité de jouer le rôle d'intermédiaire culturel entre sa propre culture et la culture étrangère et de gérer efficacement des situations de malentendus et de conflits culturels;

4. la capacité à aller au-delà de relations superficielles stéréotypées.

5 Avec la diffusion du CECR dans les politiques linguistiques éducatives, la «perspective actionnelle » tend à devenir le nouveau paradigme méthodologique de référence en didactique des langues aujourd'hui. Pour Puren (2002: 56), l'histoire des méthodologies d'enseignement des langues peut se lire à travers les recherches d'homologie successives entre les fins, «à savoir les actions que l'on veut que les élèves soient capables de réaliser en langue étrangère dans la société », et les moyens, "à savoir les actions qu'on fait réaliser aux élèves en classe ». Ainsi (ibid. : 62), pour lui, si l'approche communicative vise à former " un "communicateur" en créant des situations langagières pour le faire parler avec des interlocuteurs", dans la perspective actionnelle en revanche, le but recherché selon lui est de "former un "acteur social" » en le faisant " agir avec les autres pendant le temps de son apprentissage en lui proposant des occasions de "co-actions" dans le sens d'actions communes à finalité collective». C'est la raison pour laquelle il propose de remplacer le concept d'interculturalité par celui de co-culturalité afin de souligner le changement de perspective, la nécessité d'agir ensemble et non plus seulement celle d'établir le contact et de cohabiter en assumant nos différences. La construction européenne n'est évidemment pas étrangère à cela. Désormais en effet, tout élève doit être préparé à effectuer au moins une partie de ses études dans une deuxième langue, à se déplacer à l'étranger pour étudier et/ou pour travailler, et, sans même quitter son pays, à 
utiliser une ou plusieurs langues étrangères, notamment sur Internet, pour traiter l'information et interagir avec des interlocuteurs distants.

6 Que l'on souscrive ou non à l'utilisation du concept de co-culturalité, il n'en reste pas moins que le changement de paradigme en didactique des langues est une réalité : il ne s'agit plus de concevoir des «rencontres et échanges ponctuels» (ibid.: 63) mais d'entreprendre d'authentiques tâches collaboratives entre individus de langues et de cultures différentes.

\section{L'interculturel dans l'interaction}

7 Pour situer la dimension interculturelle dans l'interaction et en préciser le rôle, Dausendschön-Gay et Kraft (1998: 93-94) préconisent le recours à «une analyse conversationnelle rigoureuse et exhaustive [...] selon les méthodes [...] des ethnométhodologues et de la linguistique textuelle [pour] reconstruire la signification des activités communicatives dans la perspective des interactants ». Ces auteurs, fidèles à leur "position interactionniste » (ibid. 96), se proposent d'identifier dans l'échange les traces, explicites aux yeux des interactants, de «divergences d'interprétation dues à des arrière-plans linguistico-culturels différents ». À cet effet, ils distinguent deux niveaux de «manifestations d'altérité », avec une portée locale ou avec une portée globale. Dans le premier cas il s'agit d'introduire la dimension interculturelle pour résoudre un problème qui se pose localement et assurer la continuité de l'interaction; dans le second cette dimension « devient constitutive pour la définition globale de la situation » (ibid. 105) et la réalisation de tâches.

8 Les extraits cités par les auteurs pour exemplifier le premier cas sont tirés de deux corpus différents d'interactions monolingues en français où certains locuteurs sont non-natifs. L'analyse des exemples montre que la dimension interculturelle est parfois introduite pour apporter une explication, justifier un acte de parole (par exemple une question) ou un comportement, et «éviter une difficulté d'intercompréhension possible » (ibid. : 98). Plusieurs procédés sont ainsi identifiés, comme par exemple :

- un procédé contrastif, quand un locuteur désigne « le groupe auquel il appartient (chez nous, ici en France) pour classer le fait qu'il mentionne comme particulier à ce groupe » (ibid. : 99) ;

- une présentation explicitée, à l'attention de l'alloglotte, d'un « savoir que l'on présume partagé par les membres du groupe culturel ».

9 Pour exemplifier le second cas, les auteurs s'appuient sur « une rédaction conversationnelle ", en l'occurrence un échange entre une étudiante allemande et une étudiante française à qui la première demande de l'aide pour peaufiner une lettre en français à partir de sa première esquisse. Ils montrent ainsi que, dans ses corrections et suggestions, l'étudiante française se réfère à une "norme culturelle » (il faut faire des paragraphes, le $1^{e r}$ pour introduire le sujet, le $2^{e}$ pour exposer ce que tu veux,etc.) acceptée pour toute la durée de l'échange par les deux interactantes. L'interculturel est donc bien ici intégré comme "élément constitutif de la tâche » (ibid. : 107) et a une influence directe sur l'agir ensemble.

Cadre d'analyse et méthodologie

11 Sur la base des références exposées, nous distinguerons dans notre analyse trois types de manifestations de l'interculturalité : 

l'interculturel »), soit, le plus souvent, une démarche contrastive partant d'un fait, d'une loi, d'une situation identifiée sur un territoire donné (région, état...), qui conduit à des comparaisons avec les autres cultures représentées ; interculturels posés par le CECR, ceux-ci renvoyant aux fonctions de l'interculturel, ceuxlà aux capacités du sujet. Il semble bien toutefois que le type 1 soit à rapprocher des capacités 1 et 2 du CECR dans la mesure où celles-ci sont basées sur la mise en relation des cultures et l'établissement du contact avec les personnes.

16 Sur le plan méthodologique, nous prendrons appui, pour illustrer et discuter cette catégorisation, sur un choix sélectif de données tirées du forum et des clavardages des différentes sessions de formation à l'intercompréhension en langues romanes ${ }^{1}$ tenues sur Galanet, soit un volume d'archives d'environ dix mille messages pour le forum ${ }^{2}$ et de quelque trois cents clavardages d'au moins une page émis par plus d'un millier de participants - majoritairement des étudiants - issus d'établissements argentins, belges, brésiliens, français, espagnols, italiens et portugais. Ces archives ont fait l'objet d'une lecture extensive selon le «principe d'observation par balayage » (Atifi, Gauducheau et Marcoccia, 2005) de façon à extraire des exemples qui nous paraissent représentatifs des différents types. Sur le plan quantitatif, cette sélection et notre propre participation à la plupart des sessions nous permettent de faire quelques appréciations de la fréquence des phénomènes décrits, sans valeur statistique évidemment.

17 On rappellera brièvement que chaque session s'articule autour d'un scénario chronologique en quatre phases conduisant, après avoir choisi une thématique de travail, à la réalisation collaborative d'une publication plurilingue en ligne, le « dossier de presse ${ }^{3}$ ».

\section{L'omniprésence des thématiques interculturelles}

\section{Attrait de l'interculturalité}

18 Le potentiel interculturel d'un tel dispositif de formation génère une forte motivation. C'est ce qui est apparu dans les différentes enquêtes que nous avons menées auprès de plusieurs groupes d'étudiants (Degache, 2006a: 1023, Degache et Masperi, $2007: 263$ ). Ainsi, parmi une vingtaine de choix possibles, les facteurs relevant de l'attrait interculturel du dispositif (créer des liens, ouverture culturelle, enrichissement mutuel, développement d'une citoyenneté européenne) apparaissent en très bonne place. Et les résultats obtenus auprès de différents groupes réellement investis dans une formation à l'intercompréhension ont confirmé le rôle joué par cet atout interculturel, que ce soit juste avant le début d'une session ou au terme de celle-ci (Degache et Masperi, 2007 : 264).

19 La dernière enquête, menée auprès des étudiants au terme de la session «L'art du dialogue » au $1^{\text {er }}$ semestre $2006^{4}$, confirme cette tendance. Les étudiants ayant répondu 
considèrent, toujours parmi une vingtaine de choix possibles, que leur première motivation initiale était de faire une expérience d'« apprentissage sans frontières », dans un cadre international. Ce qui peut être vu, au moins en partie, comme une motivation interculturelle. Plus explicitement encore, ils placent en cinquième position «le questionnement et l'évolution de mes représentations de ces langues, de leurs locuteurs et de leurs cultures » et en septième position « la découverte des aspirations, des modes de pensée, des valeurs des locuteurs d'autres langues romanes ». Ces motivations sont consolidées au fil de la session, pour atteindre un taux de près de 3 sur une échelle de 4 , même si d'autres motivations surgissent, voire les dépassent (à noter par exemple la forte progression des motivations de nature cognitivo-langagières ${ }^{5}$ et socio-affectives $\left.{ }^{6}\right)$.

\section{Confirmation des motivations}

20 Les motivations interculturelles des participants sont confirmées dans les interactions et les produits des tâches et sous-tâches de la session. Elles apparaissent dès son ouverture, dans les profils complétés par les participants (voir ici même Araújo e Sá et al.) et dans des messages exprimant l'envie de découvrir et de partager les cultures: "Espero poder transmitirles algo de nuestra cultura y hacerles conocer nuestro pais [J'espère pouvoir vous transmettre quelque chose de notre culture et vous faire connaitre notre pays] $]^{7}$ [Nora, CA_050304] ; parfois même dans des langues romanes non ciblées expressément comme ici en roumain : "Românca de origine si de limba materna, va invit sa descoperiti limba romana care vine dintr-o "mare slava", din Estul Europei [...] bun demaraj [Roumaine d'origine et de langue maternelle, je vous invite à découvrir la langue roumaine qui vient de la “mer slave" de l'est de l'Europe [] bon démarrage!] » [NadiaM, VP_010207].

Ce désir d'interculturalité est encore plus prégnant dans les thématiques des sujets proposés dès la première phase du scénario où il s'agit de faire des propositions en vue de choisir un thème pour la tâche collaborative collective. Ces sujets peuvent être classés en cinq catégories :

a. métacommunicatifs : « La chat come strumento di comunicazione...!!! » AD06

b. universels : concernant des questions à l'échelle mondiale («o cambio climático » VP07), des questions ou faits «extra-romans » («La crise au moyen orient » VP07) ou encore des comparaisons interpersonnelles de nature métacognitive ( $«$ ma voi... come studiate ??? [mais vous... comment étudiez-vous ?] » AD06) ou socio-affective (« Esiste ancora la vera amicizia ? [La vraie amitié existe-t-elle encore ?] » FP06) ;

c. métalangagiers : «El contacto entre culturas ¿enriquece o empobrece la lengua? [Le contact entre les cultures enrichit-il ou appauvrit-il la langue ?] » AD06 ;

d. explicitement interculturels : proposant d'interroger les stéréotypes («Stéréotypes: ouverture ou fermeture aux autres cultures?» LC07) ou de parler ouvertement d'interculturalité (« La inter-culturalidad juvenil » AD06), ou encore de voir ce qui unit ou sépare les différentes cultures représentées ("¿Qué une y qué separa a las diferentes culturas? » VR05);

e. contrastivement interculturels: proposent une approche contrastive sur un fait socioculturel donné (par exemple le mariage homosexuel, la formation universitaire, l'interdiction de fumer dans les lieux publics...) ou bien ce qui représente le mieux chaque culture sur un point donné (livre, film, mets, chanson...).

Dans une estimation établie par ailleurs (Degache, 2006a), $50 \%$ des sujets proposés correspondent aux deux dernières catégories et peuvent donc être considérés comme 
interculturels. Néanmoins, cette classification étant réalisée à partir du titre et de la présentation du sujet proposé («l'amorce»), il faut garder à l'esprit, sur la base de la distinction en trois types que nous avons posée plus haut, que : - tout sujet, y compris un sujet universel, peut à tout moment faire l'objet de l'introduction de la dimension interculturelle dans l'interaction. On pourrait alors dire, sur le modèle de l'exolinguisme, qu'il devient «exoculturel», c'est-à-dire que le différentiel culturel est assumé et pris en charge par au moins un locuteur pour poser un élément explicatif propre à sa culture de référence ;

24 - les interactants semblent rester en permanence conscients du contexte de « rencontre interculturelle » (De Nuchèze, 2004) que constitue la session. Preuve en est que nombre de sujets et d'échanges portent, au-delà de la parenté linguistique, sur la recherche d'une parenté culturelle entre locuteurs de langues romanes, soit en définitive sur la nécessité/ difficulté de se constituer en tant que communauté : «Pourquoi êtes-vous ici? »; «La diversidad lingüística ¿nos une? ¿nos separa? [La diversité linguistique nous unit-elle ou nous sépare-t-elle?]»; "Qui sont-ils ces Néo-Latins dans le regard de l'autre?» LC07. Recherche qui se concrétise dans l'utilisation du déictique "nous" qui peut être considéré comme « un indice de l'existence d'un sentiment communautaire» (Carton, 2006:21). On en prendra pour preuve le sujet «La "romanophonie" » dans la session FP06, présenté ainsi :

Quels sont les points communs entre romanophones? Mise à part nos langues romanes quels sont nos principaux points communs dans nos pays, cultures, mentalités, vies quotidiennes... Souffrons-nous des mêmes clichés les uns les autres?

\section{Les savoir-faire concernés}

25 À travers les thématiques proposées et finalement retenues et développées au fil des sessions - on notera que six des huit «dossiers de presse » publiés font peu ou prou explicitement référence à la dimension interculturelle dans leur titre ou leur chapeau -, les habiletés interculturelles pratiquées relèvent principalement des deux premiers savoir-faire identifiés par le CECR (cf.supra), moyennant une adaptation au contexte pluriculturel de la situation :

26 - la capacité à mettre en relation la (ou les) culture(s) d'appartenance et les cultures étrangères se fait certes autour des cultures des pays et régions de langues romanes mais elle peut concerner également des cultures " extra-romanes ", soit à travers certaines thématiques, soit en tenant compte du fait que nombre de participants possèdent des cultures autres (étudiants africains, arabes, germaniques, slaves, scandinaves...). Cette habileté se manifeste de surcroit avec le souci de prendre en compte la diversité régionale et sociale des mondes représentés et «la manière dont chaque communauté apparaît dans l'optique de l'autre » (CECR, p. 83);

27 - la capacité de reconnaitre et d'utiliser des stratégies variées pour établir le contact avec des gens d'une autre culture se réalise par des références à des connaissances préalables ou à l'expérience vécue en personne dans l'autre culture, ou encore par des questions contrastives, parfois à fond polémique pour s'assurer d'une réaction. C'est sur ces aspects potentiellement ou effectivement conflictuels que nous allons poursuivre l'analyse. 


\section{La gestion des tensions}

28 CECR appelle l'«intermédiaire culturel» dans le troisième savoir-faire, on relève toutefois ponctuellement dans les sessions quelques tensions interculturelles. On en examinera ici trois exemples.

Dans la première séquence, un chat (VR 160305, salon bleu), le conflit culturel potentiel que pourrait introduire la thématique «les Français sont un peu fermés » par Luiza, Brésilienne, va être évité du fait des réactions de ses interlocutrices :

\begin{tabular}{|l|l|}
\hline [Luiza] Sonia... on disait que les Français & \\
\hline sont un peu fermés [...] & \\
\hline [Sonia] acham mesmo que os franceses & Vous trouvez que les \\
\hline são fechados [...] & Français sont fermés... \\
\hline [ChantalM] je pense ça aussi & \\
\hline [Sonia] porquê ???? [...] & \\
\hline [Sonia] o que é ser fechado ??? & C'est quoi être fermé ??? \\
\hline [ChantalM] enfin surtout dans mon & \\
\hline département, car je vis à la frontière & \\
\hline suisse et les gens sont obsédés par & Timide ce n'est pas la même chose... \\
\hline ['argent liui donnent ! \\
\hline [Sonia] Mas, o que entendem por ser & Mais qu'entendez-vous \\
\hline fechados???? & par être fermés ???? \\
\hline [ChantalM] êtres fermés [...] le même & \\
\hline [Sonia] ser fechado pode significar & Étre fermé peut signifier timide... \\
\hline Tímido.... e não me parece francês no & \\
\hline \hline
\end{tabular}




\begin{tabular}{|l|l|}
\hline [ChantalM] ben non ce nest pas du & \\
\hline tout pareil & \\
\hline [ChantalM] voyons un peu!!! [...] & \\
\hline [Luiza] mas as pessoas fechadas podem & Mais les personnes fermées \\
\hline não ser tímidas.... je trouve pas q les & peuvent ne pas être timides... \\
\hline Français soient timides... loin de ça [...] & \\
\hline [ChantalM] je suis d'accord avec toi!! & \\
\hline tout dépend de la personnalité de chacun, & \\
\hline cela de vient pas de la nationalité & \\
\hline
\end{tabular}

Dans cet échange exolingue-bilingue, Sonia, portugaise, par la question qu'elle pose sur le sens de l'expression "être fermé/ser fechado ", invite Luiza et Chantal à interroger le stéréotype de départ (capacité 4 du CECR), même si Chantal, la seule Française de l'échange, l'a validé par un témoignage tiré du vécu personnel (tout en l'attribuant implicitement à une influence étrangère).

31 La deuxième séquence débouche sur une légère divergence de point de vue quant à la manière d'apprécier les stéréotypes culturels, mais, comme ci-dessus, la volonté de les dépasser y est également attestée. L'étendue de la gamme des cultures représentées sur Galanet le permet facilement. Dans cet échange en espagnol entre KatiaK (étudiante Erasmus polonaise de l'équipe de Madrid) et Angelica (étudiante colombienne de l'équipe de Grenoble) dans le sujet "Stéréotypes : ouverture ou fermeture aux autres cultures?» LC07. Du fait du positionnement de chacune des interactantes (« como colombiana te digo... »; " tú eres colombiana, yo soy polaca... »), suite à l'émission spontanée de la part de KatiaK d'un avis personnel qu'Angelica ne partage pas ( il faut bien accepter les stéréotypes en les prenant avec humour »), l'échange passe subitement du type 1 (une évocation distanciée des stéréotypes les plus courants sur les différentes nationalités représentées) au type 2 (une mobilisation des données interculturelles pour s'engager personnellement) : 


\begin{tabular}{|c|c|}
\hline $\begin{array}{l}\text { KatiaK [LC_120307] [...] tenemosque aceptar que los } \\
\text { estereotipos yase han arraigado en nuestrasculturas } \\
\text { y no hay más remedio quetomarlos todos con cierta } \\
\text { dosis delhumor. } \\
\text { Angelica [LC_150307] : KatiaK alleer tu mensaje me } \\
\text { doy cuenta deque nunca has sufrido por } \\
\text { losestereotipos... como colombiana tedigo que es } \\
\text { difícil de tolerar cuandocada vez que le dices a la } \\
\text { gente dedonde vienes lo asocianinmediatamente a } \\
\text { la droga o a laviolencia... [...]... es mucho más } \\
\text { fácilcuando el estereotipo es el futbol, lacomida o la } \\
\text { fiesta... o tal vez la faltade ducha... pero ¿qué hacer } \\
\text { con losque duelen ?... } \\
\text { KatiaK [LC_150307] Sí que losestereotipos pueden } \\
\text { hacer daño, nolo niego y yo también he sufrido } \\
\text { porellos en su momento. Tu erescolombiana, yo soy } \\
\text { polaca y mí paísen la mayoría de los } \\
\text { europeostampoco despierta asociacionespositivas. } \\
\text { Bueno, digamos quealgunos piensan que lo que } \\
\text { solemoshacer es beber vodka y robar cochesde los } \\
\text { alemanes que vienen devisita... [...] }\end{array}$ & \begin{tabular}{|l} 
Nous devons accepter que les stéréotypes sont \\
déjà enracinés dans notre culture et qu'il n'y \\
a pas d'autre remède que les prendre tous \\
avec une certaine dose d'humour. \\
KatiaK, en lisant ton message je me rends \\
compte que tu n'as jamais souffert des \\
stéréotypes... en tant que Colombienne je te \\
dis que c'est difficile à supporter quand \\
chaque fois que tu dis aux gens d'où tu viens \\
ils l'associent immédiatement à la drogue ou \\
la violence [...] c'est beaucoup plus facile \\
quand le stéréotype c'est le football, la \\
nourriture ou la fête... ou peut-être le manque \\
de douche... mais que faire avec ceux qui \\
blessent? \\
Bien sûr que les stéréotypes peuvent blesser, \\
je ne le nie pas et moi aussi il m'est arrivé \\
d'en souffrir. Tu es colombienne, je suis \\
polonaise et mon pays pour la majorité des \\
Européens n'éveille pas non plus des \\
associations positives. Bon, disons que \\
certains pensent que nous avons l'habitude de \\
boire de la vodka et de voler les voitures des \\
Allemands qui nous rendent visite... [...]
\end{tabular} \\
\hline
\end{tabular}

32 La troisième séquence, également dans un forum, débouche pour sa part sur une réelle «situation de malentendu et de conflit culturel» (CECR, p. 84). Il s'agit d'un sujet présenté par Ioana, étudiante roumaine de Lyon, avec une orientation polémique sous le titre «La grève est-elle une spécialité française?» (AD06) au moment du mouvement social du printemps 2006 en France :

Ioana : En France tout les jours il y a une grève. En Lyon il y a aussi presque toutes les semaines une grève pour les milieux de transports. J'essaie de m'habituer à la situation, mais pour moi comme je suis étudiante étrangère, ce n'est pas très facile. Enfin, je n'arrive pas à comprendre cette habitude française. Avez une réponse pour moi ?

Les premières réactions cherchent à placer le débat sur un plan universel et non sur un plan interculturel :

CorrineA: Je ne crois pas que l'on puisse dire que la grève est une "habitude française". La grève rentre dans les différentes manières de dialoguer avec le gouvernement pour refuser ce qu'il propose. Et toutes les démocraties qui se respectent dialoguent avec leurs gouvernements et descendent dans la rue Timge : [la France] est loin d'être le pays qui réussit à perdurer les grèves les plus longtemps possible. (Je vous rappelle par exemple les Vénézuéliens qui ont fait plus de 6 mois de grèves il y a 3-4 ans de ça.)

De nombreuses réactions se livrent à des comparaisons avec la situation dans d'autres pays, réalisant ainsi des réflexions interculturelles de type 1 , dont on peut dire, en raison de leur orientation argumentative - explicite ou implicite -, qu'elles tendent vers des prises de position ${ }^{8}$ de type 2 : 
Anetta: [...] credo che in Francia tutte le manifestazioni siano più efficaci, almeno per quanto riguarda l'organizzazione e il sentimento di "protesta"... qui da noi è sempre tutto troppo confusionario frammentario... e a volte le persone si adagiano su questo!

MDoloresB : [...] mientras que los estudiantes franceses luchan por su futuro, los españoles, como habréis podido ver en los medios de comunicación, luchan por ser losprimeros en las botellonas

Eva_D : Aqui no Brasil temos sempre alguma greve no sistema de ensino... mas essas não são como as greves francesas, porque praticamente não há manifestações! (italien) je crois qu'en France toutes les manifestations sont plus efficaces, au moins en ce qui concerne l'organisation et le sentiment de "protestation "... ici chez nous tout est toujours trop confus et fragmenté... et parfois les gens se fixent là-dessus!

(espagnol) pendant que les étudiants français luttent pour leur futur, les Espagnols, comme vous aurez pu voir dans les médias, luttent pour être les premiers dans les botellones (beuveries de rue)

(portugais) Ici au Brésil les grèves sont fréquentes dans le système éducatif... mais elles ne sont pas comme les grèves françaises, parce qu'il n'y a pratiquement pas de manifestations!

Le débat collectif plurilingue amène une étudiante à demander des précisions :

Nelly : [...] comment ça se passe en Roumanie quand les gens sont opposés à une mesure prise par le gouvernement. Est-ce qu'ils font grève? Est-ce qu'ils se taisent ? Est-ce qu'ils font entendre leur opinion autrement?

Ce à quoi Ioana répond en mobilisant des données interculturelles avec une finalité clairement argumentative (type 2) :

Ioana : Nelly, il y a partout des grève et je trouve ca normale! Mais pas partout de la grève exagérée. Si quelqu'un est mecontent, il va protester, mais tout est mesuré. [...] La dernière grève pour les trains et métros en Romanie il y avait un an et demi et ca a durée que deux heures. Crois-moi, en Roumanie les gens ont trouvé un vrai raison d'organiser cette grève.

Si bien que l'échange prend une orientation socio-politique où Ioana se retrouve seule contre tous. Ainsi lorsque Karim écrit «La tradition française à la grève remonte à la révolution française de $1789 . .$. ", elle répond " Escuse moi, Karim si te contredirai un peu mais tu est un peu trop centré sur la France [...] en plus les Francais n'ont pas du tout inventé la greve !!!! », ce qui conduit à des prises de position de la part des autres participants (type 2) où l'on voit apparaitre des représentations plus marquées de la culture de l'autre et soutenant le mouvement de protestation et le droit de grève : 
stefania: [...] credo che latradizione rivoluzionaria francesenon abbia pari e sembra che ifrancesi ne conservino gelosamentetracce nel loro dna... [...]feirense : [...] os franceses sãomuito revolucionários, as grandesrevoluções aconteceram em França, e ainda hoje se manifestamconstantemente quando não estãosatisfeitos com algo! mas eu pensoque é assim que devem ser, pois nãodevem nunca deixar de lutar pelosseus direitos ! [...] (italien) je crois que la tradition révolutionnaire française n'a pas d'équivalent et que les Français en conservent jalousement des traces dans leur ADN.

(portugais) les Français sont très révolutionnaires, les grandes révolutions se sont produites en France, et aujourd'hui encore ils manifestent constamment quand ils ne sont pas satisfaits de quelque chose! mais moi je pense qu'il doit en être ainsi puisqu'il ne faut jamais cesser de lutter pour ses droits!

Au total, en deux jours, six messages de la part de 6 participants non français (3 Portugais, 2 Italiens, 1 Turc) issus de 4 équipes différentes, écrits en 3 langues (it, fr, po), s'opposent de quelque manière à Ioana. Aucun message pour la soutenir en revanche. Face à cette unanimité plurilingue et pluriculturelle, elle réagira en roumain par des paroles très fortes :

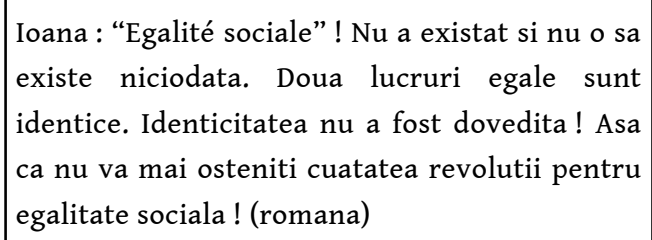

(roumain) L"Égalité sociale"! Elle n'a pas existé et elle n'existera jamais. Deux choses égales sont identiques. L'identité-égalité n'a jamais été démontrée. Donc ne vous donnez plus de peine avec tant de révolutions pour l'égalité sociale!

On peut voir là une alternance codique ambivalente, à la fois convergente et divergente. Convergente parce que Ioana affirme ainsi son identité romanophone, divergente parce qu'elle le fait sous une formulation difficile à appréhender sans traduction'. Presque une sorte de fin de non recevoir à la suite de quoi l'échange ne progressera plus beaucoup.

Néanmoins, le fond de polémique présent dans ce sujet de discussion reste une exception contrairement aux pratiques relevées dans des forums publics non-pédagogiques où l'invective est souvent la règle, le plus souvent sous couvert d'anonymat. Au fil des sessions, le contexte pédagogique et une perception positive du principe d'intercompréhension, sans doute aussi la présence sur le site d'une charte fixant les règles de bonne conduite, font que les sources potentielles de conflit sont en général gommées.

\section{La co-culturalité dans l'interaction plurilingue}

41 Les manifestations interculturelles précédemment mentionnées relèvent des types 1 et 2 . Il y a donc lieu de se demander si, dans le scénario Galanet, la dimension interculturelle peut être mobilisée pour autre chose que pour un échange d'opinions. En réalité, le type 3 est relativement rare, ce qui nous amène à faire le constat que, en définitive, il y a peu de 
traces de co-culturalité dans les échanges. Comment peut-on expliquer cela? Faut-il y voir :

- un faible potentiel « co-culturel » du scénario de la plateforme?

- un effet de la « culture partagée » des participants ?

- ou, au contraire, peut-on avancer que les traces de « co-culturalité » existent bien mais qu'elles se dissimulent dans un ensemble plus vaste?

\section{Scénario et potentiel « co-culturel »}

Le peu de traces de co-culturalité dans les interactions peut s'expliquer par le rôle central joué par le forum dans la formation. Cet outil, de par ses spécificités, notamment par sa structuration relativement figée qui oblige à rendre explicites les réactions à un message particulier au sein d'un sujet de discussion ${ }^{10}$, pourrait favoriser l'émergence des manifestations interculturelles de types 1 et 2 . Surtout, la nature des tâches intermédiaires que l'on est invité à y réaliser, pourrait expliquer leur prédominance. En effet, il s'agit de proposer des sujets, de débattre de leur potentiel, d'argumenter sur leur intérêt, puis une fois que l'un d'entre eux a été choisi, d'en dégager une série de sousthèmes puis de débattre autour de ces sous-thèmes à partir de documents d'appui fournis ou produits par les participants avant d'arriver, in fine, à la réalisation de la tâche finale, le «dossier de presse». Certes, au moment de l'élaboration des rubriques dans cette dernière phase, cette co-culturalité devrait être favorisée. Mais en raison d'un certain nombre de limitations du dispositif de formation, tant du point de vue du scénario d'encadrement pédagogique que de l'environnement informatisé, en particulier au niveau de l'outil de réalisation du dossier de presse et de ses fonctionnalités, cela n'est pas le cas. En effet, la mise au point de ces rubriques à partir de synthèses des échanges antérieurs et des documents d'appui, ne se fait pas en l'état actuel des choses de manière suffisamment collaborative entre participants de différentes équipes mais plutôt au sein d'une même équipe. Si bien qu'au niveau de la dernière phase de la session, l'interaction plurilingue souffre en général d'une baisse de rendement, l'interculturalité - et la coculturalité - également par voie de conséquence. Non pas que l'outil en empêche la mise au point mais ce n'est qu'au prix d'une organisation spécifique et d'un tutorat renforcé que cela peut être effectivement possible, ce qui a peu été fait jusqu'à présent ${ }^{11}$.

\section{Effets possibles d'une culture partagée}

Définir l'univers culturel d'un sujet renvoie à une complexité dont il faut tenir compte pour évaluer la notion de culture partagée. D'une part, tout sujet construit son propre univers culturel en fonction de la culture de la communauté linguistique à laquelle il appartient et de son niveau socioculturel, ce qui suppose une diversité de connaissances partagées à l'intérieur de chacune des communautés linguistiques, le fonds commun restant à définir mais se présentant comme le composant de l'identité de la communauté. D'autre part, la composante socioculturelle offre des similitudes entre groupes d'une communauté linguistique à une autre, ces connaissances partagées entre individus socioculturellement positionnés leur confèrent une valeur de communauté culturelle homogène sous bien des aspects.

Pour mener à bien notre analyse, nous devons tenir compte de ces deux paradigmes qui peuvent se succéder dans un même forum ou même se cumuler dans une même 
interaction dans des mouvements différents. Cependant on peut souligner l'homogénéité de l'univers culturel qui caractérise les participants sur la plateforme Galanet aussi bien socialement que technologiquement.

\section{Une homogénéité linguistique et sociale}

Les participants à la plateforme Galanet présentent en effet beaucoup de points communs. Beaucoup sont étudiants spécialistes de langues ou de linguistique et de didactique, ce sont donc des sujets ayant au niveau culturel une formation et des centres d'intérêt concordants. Leur motivation est elle-même similaire, ils s'intéressent à l'apprentissage des langues, tant pour les apprendre que pour les enseigner, et ils sont formés à une réflexion sur les langues et le langage, ce qui donne à leurs stratégies une dimension théorique et métalinguistique que l'on ne retrouverait pas à cette hauteur si le profil des participants était différent.

Cette formation initiale se complète par un accès à des sources d'information - journaux, TV, net, cinéma, publicité, etc. - similaires, ce qui accentue leur appartenance a un groupe d'âge homogène qui leur procure des références toujours plus proches dans des sociétés qui tendent à la globalisation et effacent les différences nationales, en particulier en Europe.

Dans l'ensemble, les milieux sociaux auxquels ils appartiennent sont apparentés d'un pays à un autre et ils partagent des projets d'avenir similaires, les études qu'ils ont choisies offrant des débouchés professionnels semblables dans les différentes sociétés.

Bien évidemment les étudiants des différentes équipes de Galanet sont des locuteurs des langues romanes soit parce que c'est leur langue maternelle ou leur première langue de scolarisation soit, surtout dans le cas des Erasmus, parce qu'ils ont un haut niveau de compétence linguistique en langues romanes. Un bon nombre d'entre eux connaissent une ou deux autres langues de la même famille.

\section{De quelle culture peut-on parler?}

49 Aborder la notion de culture est beaucoup moins simple. Certes, on peut considérer que les cultures des pays latins partagent un socle commun de valeurs, de croyances et de connaissances; il n'en reste pas moins que chacune des cultures garde une identité propre, même si la langue est commune, comme c'est le cas de l'Espagne et de l'Argentine ou du Portugal et du Brésil, et que ces différences sont constitutives de l'identité nationale des participants.

Cependant nous considérons que toutes les connaissances construites par chacun des individus au cours de sa formation - voir paragraphe précédent - confèrent une homogénéité à l'ensemble des participants qui lui permet d'affirmer son identité nationale à partir de la position qui les unit en tant que groupe socioculturel. C'est pourquoi les controverses et les stéréotypes sont relativement peu nombreux dans les forums.

\section{Une co-culturalité dissimulée?}

Bien qu'il y ait peu de traces de co-culturalité - du «dire pour agir» - dans les interactions, on les voit parfois apparaitre néanmoins au moment des prises de décisions 
qui engagent les étudiants à certains moments du scénario. C'est notamment le cas dans un chat (salon rouge, 070305) portant sur l'intérêt comparé des thèmes proposés en vue du vote en phase 1 . Un étudiant barcelonais souligne l'intérêt du thème métalangagier «Binomio idioma/país. ¿Corresponden las fronteras de un país a la representación y a la utilización de un idioma? [Binôme langue/pays. Les frontières d'un pays correspondent-elles à la représentation et à l'utilisation d'une langue ?] " compte tenu des différences culturelles sur la question, notamment chez les équipes françaises et espagnoles. L'argument interculturel est bien convoqué ici pour peser sur l'action, chose d'ailleurs confirmée immédiatement par l'intervention suivante: "(Los catalanes siempre defendemos lo nuestro en grupo) jeje », soit une parenthèse auto-ironique, « jeje » étant équivalent au sourire complice ;-)

Dans le même chat un peu plus loin, entre trois autres étudiants (une Française et deux Espagnols), on trouve la séquence suivante :

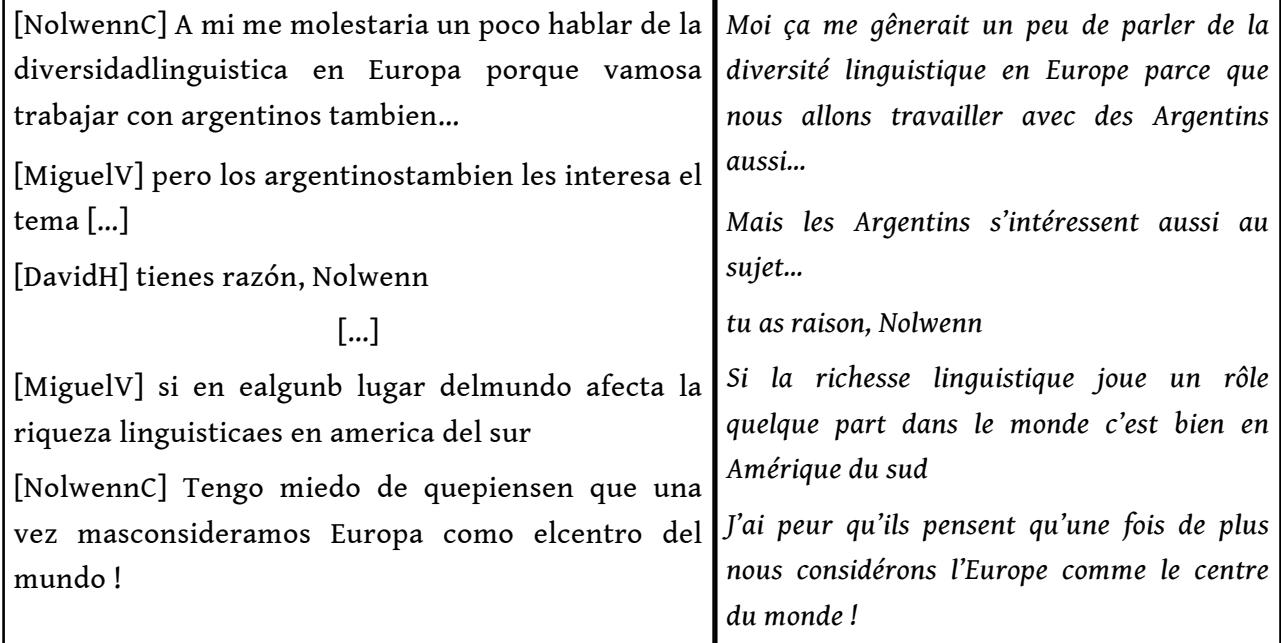

Moi ça me gênerait un peu de parler de la diversité linguistique en Europe parce que nous allons travailler avec des Argentins aussi...

Mais les Argentins s'intéressent aussi au sujet...

tu as raison, Nolwenn

Si la richesse linguistique joue un rôle quelque part dans le monde c'est bien en Amérique du sud

J'ai peur qu'ils pensent qu'une fois de plus nous considérons l'Europe comme le centre du monde!

Cette fois, les trois participants mobilisent connaissances et représentations culturelles sur un autre groupe de participants, les Argentins, pour peser le pour et le contre du choix d'un sujet (leur vote en réalité) qui pourrait être trop européo-centré. C'est la question du rapport entre identités romanophone et européenne qui est alors posée.

\section{Conclusions}

La présente étude a permis d'aboutir à une catégorisation en trois types de manifestations interculturelles. L'identification et la discussion d'un certain nombre d'exemples nous permet de mieux en appréhender la portée et les spécificités et il peut être envisagé, dans les sessions à venir, de s'inspirer de cette catégorisation pour mieux apprécier le type d'interculturalité en jeu dans les échanges.

L'analyse que nous avons conduite ici porte à conclure que le concept d'échange mis en œuvre sur cette plateforme, basé sur l'interaction plurilingue dans la famille des langues romanes entre locuteurs d'origines diverses maitrisant au moins une langue romane en tant que langue 1 ou 2, est propice à la rencontre interculturelle. Ils y voient là un attrait majeur du dispositif comme on a pu le vérifier. Dans chaque session on retrouve, en 
général au début, des thèmes récurrents portant sur un questionnement identitaire (un des plus parlants est le $1^{\text {er }}$ sujet de VR05 «Quem somos e para onde vamos ?: O que nos une nesta sessão? Quais são os nossos objectivos ? Onde estamos? Até onde queremos ir ? [Qui sommes-nous et où allons-nous? : qu'est-ce qui nous unit dans cette session? Quels sont nos objectifs? Où en sommes-nous ? Jusqu'où voulons-nous aller ?] »). Fréquemment, on peut noter que ce questionnement conduit les participants à se définir comme une communauté de non-anglophones (Carton, $2006: 21)$ et à s'interroger sur la relation entre communauté européenne et communauté romanophone, relation vue dans un premier temps comme inclusive mais vite remise en question du fait notamment de la présence d'équipes latinoaméricaines et de participants originaires du continent africain.

Nous avons ainsi montré que le dispositif est particulièrement adapté à l'exercice des capacités et à la prise de conscience interculturelles (CECR: 83-84), car il facilite l'interrogation des attitudes et valeurs personnelles associées aux cultures, par leur mise en contraste avec celles des autres participants comme l'exprime cette étudiante de Lyon lorsqu'elle soumet au vote en phase 1 un sujet intitulé "L'interculturalité » libellé ainsi «[...] dans interculturalité, au-delà du simple échange entre personnes venant de différentes cultures, je ressens un sens plus profond, qui est de creuser en soi-même ; et ceci parfois grâce à autrui, qui peut ou non venir d'une autre culture ».

En revanche, en l'état actuel des choses, l'exercice de la co-culturalité ne semble pas réellement favorisé dans la mesure où les occasions d'agir ensemble en ligne et en plusieurs langues, restent limitées, alors même qu'elle peut être considérée comme la plus favorable au développement de l'interaction et des compétences d'intercompréhension plurilingue (Masperi et Quintin, à paraitre). Cette discrétion relative peut s'expliquer, au moins en partie, par un effet de culture partagée entre étudiants: inutile de convoquer des données culturelles dans l'action et la prise de décision si on considère ou constate que la culture des interactants est fort semblable. Toutefois, au vu des quelques exemples que nous avons identifiés ici, il semble probable que les fonctionnalités de certains outils, ou autrement dit les modalités de réalisation de la tâche, soient également responsables de cet état de fait, ce qui pourrait être considéré comme une "affordance» (voir à ce sujet Mangenot, $2007: 6$ ). Si cela est confirmé, le dispositif de formation devra d'abord évoluer. Ce n'est qu'alors, lorsque les étudiants des différentes équipes seront plus impliqués dans la réalisation de tâches collaboratives en ligne, de manière synchrone ou asynchrone, que nous pourrons apprécier réellement la co-culturalité. Observera-t-on alors différentes cultures d'apprentissage, par exemple dans une tâche d'écriture collaborative comme la synthèse d'un forum puisque c'est un genre pratiqué dans le «dossier de presse", autour de questions comme: comment la structurer, comment rapporter le discours d'autrui, que garder ou quel rapport doit-il y avoir entre volume d'origine et volume de la synthèse, comment gérer le plurilinguisme ? À moins que ce type de tâche ne soit trop novateur pour favoriser la convocation de normes culturellement marquées? Comme si en définitive, contexte et situation pédagogiques étaient si différents (travail en autonomie, pédagogie du projet, travail collaboratif, type de tâches, utilisation des technologies, caractère plurilingue des échanges...) de ce qui est habituellement pratiqué, qu'aucune culture d'apprentissage particulière ne se retrouverait réellement favorisée ou désavantagée?

Mais la recherche devra également s'intéresser à d'autres types de données pour mieux appréhender la dimension interculturelle dans un tel dispositif. Il convient en effet de noter que nous n'avons considéré que les interactions en ligne et délibérément ignorées 
d'autres sources de données, comme par exemple les interactions locales au sein d'un même groupe, notamment lors des regroupements présentiels, parce que ce qui n'est pas dit publiquement en ligne est peut-être quand même très présent (comment sont commentées les tensions éventuelles? qualifie-t-on par exemple de «typiquement espagnol, français, brésilien... » tel ou tel comportement, réaction ou attitude ?). Sans doute perçus comme potentiellement polémiques et contraires au principe général de politesse interculturel, voire à la charte du participant, ils sont en général passés sous silence en ligne. Il est d'autant plus important de prêter intérêt à ces données qu'il a déjà été noté qu'il peut exister " un clivage entre les activités en situation de contact d'un côté et la façon dont les participants à ces situations en parlent à d'autres personnes » (Dausendschön-Gay et Kraft, 1998 : 108).

\section{BIBLIOGRAPHIE}

\section{Références bibliographiques}

(liens internet vérifiés le 1-09-2007)

Álvarez, S. (2006) : Interacciones sincrónicas en un contexto pedagógico virtual : análisis estructural e interaccional, Proyecto de investigación,Universitat de Lleida/Universidad Stendhal-Grenoble 3.

Atifi, H., Gauducheau, N. \& Marcoccia, M. (2005) : Les manifestations des émotions dans les forums de discussion, Journée d'étude Les émotions dans les interactions en ligne. ICAR ENS LSH, Lyon, 17 mars 2005. Consulté en septembre 2007.

Carton, A. (2006) : Communauté en ligne, discours et acquisition dans un forum de formation plurilingue, Mémoire de Master 1 Études italiennes, ENS LSH Lyon \& Université Grenoble 3.

Conseil de l'Europe (2001) : Cadre européen commun de référence pour les langues. Apprendre, enseigner, évaluer, Conseil de la Coopération culturelle, Comité de l'éducation, Division des langues vivantes, Strasbourg, Paris, Les Éditions Didier.

De Nuchèze, V. (2004) : La rencontre interculturelle. Impasses, sentiers balisés et chemins de traverse, Lidil, 29, 11-41.

Dausendschön-Gay, U., Krafft, U. (1998) : Quand l'exolingue devient de l'interculturel, Lidil, 18, Grenoble, Lidilem, 93-111.

Degache, C. (2006a) : Acercarse al otro y a su cultura : el componente intercultural de una formación en Internet para hispanohablantes, francófonos y hablantes de otras lenguas románicas, Actas del Primer Encuentro Hispanofrancés de Investigadores (APFUE-SHF), La cultura del otro : español en Francia, francés en España, Universidad de Sevilla, 1020-1032,

Degache, C. (2006b) : Aspects du contrat didactique dans une formation plurilingue ouverte et à distance, Le français dans le monde, Recherches et applications, 40, 58-74.

Degache, C., Masperi, M. (2007) : Représentations entrecroisées et intercompréhension, chapitre 25, P. Lambert, A. Millet, M. Rispail et C. Trimaille (éds.), Variations au cour et aux marges de la sociolinguistique, Mélanges offerts à Jacqueline Billiez, coll. Espaces discursifs, L'Harmattan, 259-270. 
Mangenot, F. (2007) : Pratiques pédagogiques instrumentées et propriétés des outils : le cas des forums, M.-N. Lamy, F. Mangenot et E. Nissen Actes du colloque Échanger pour apprendre en ligne ( $\underline{E P A L}$ ), Grenoble, 7-9 juin 2007,

Masperi, M., Quintin, J.-J. (à paraitre) : Modèle de scénario pédagogique pour la pratique de la compréhension croisée plurilingue à distance : élaboration, usage et effets, Actes du Colloque international Scenario 2007 - 14-15 mai 2007, Montréal, Canada,

Puren, C. (2002) : Perspectives actionnelles et perspectives culturelles en didactique des languescultures : vers une perspective co-actionnelle-co-culturelle ", Les Langues modernes, 3, 2002, APLV, Paris, 55-76.

\section{NOTES}

1. La référence aux sessions sera faite ici par deux lettres : CA canosession, SO Sessione ottobrenovembre 2004 ; VR Verba Rebus ; AD L'art du dialogue ; FP Para cada frontera hay un Puente ; LC Entre Línguas e Culturas ; VP Visión panorámica.

2. Les forums, ainsi que plusieurs autres espaces et outils, sont accessibles en tant que « visiteur » sur: Galanet, rubrique « Sessions terminées » > choix d'une session > «Visiteur ».

3. Pour plus de détails sur l'environnement d'apprentissage et le scénario d'une session, on se reportera aux articles disponibles dans l'espace «Les publications » sur : Galanet et à Araújo e Sá et al. (ici-même).

4. Enquête réalisée par questionnaire écrit délivré à distance au moyen du forum de cette session. 50 participants avec le statut "étudiant» ont répondu, chiffre à comparer aux 134 participants s'étant connectés au moins 10 fois durant la session. Il s'agissait d'évaluer, sur une échelle de 0 à 4 et pour une vingtaine de critères, le degré de motivation initial, intermédiaire et final, Consulter.

5. Notamment le fait d'avoir développé ses connaissances en situation de communication, d'avoir amélioré sa capacité de compréhension, et, surtout, d'avoir fait découvrir sa langue aux autres et les avoir aidés à la comprendre.

6. Comme une meilleure connaissance de soi-même, de ses réactions, dans une situation d'apprentissage complexe, la pratique de modalités relationnelles différentes avec étudiants et enseignants, ou encore la découverte de nouvelles modalités d'apprentissage actionnelles, en situation et avec une certaine autonomie.

7. Les extraits rapportés n'ont fait l'objet d'aucune correction. Une traduction est fournie en parallèle ou entre crochets en italique lorsque la parenté linguistique ne permet pas un accès aisé à la compréhension.

8. L'échange étant ici exolingue-plurilingue, la langue originale de l'extrait traduit est indiquée entre parenthèses.

9. On pourrait également penser qu'elle se démarque ainsi des intervenants qui n'ont pas une langue romane comme première langue (notamment Timge et Karim), mais rien d'explicite ne nous permet d'aller dans ce sens.

10. Alors que d'autres forums autorisent le développement de fils de discussion à partir de n'importe quel message initial.

11. La seule initiative en ce sens est celle, à notre connaissance, mise en place entre les équipes de Barcelone et de Grenoble dans la session Verba Rebus (Alvarez, 2006 : 31), par des chats visant à organiser le travail conjoint dans une rubrique du dossier de presse de cette session, initiative dont on trouve également des traces dans le forum. 


\section{RÉSUMÉS}

Comment les compétences interculturelles s'expriment-elles dans un environnement de formation sur Internet, réunissant plusieurs groupes d'apprenants de différents pays et utilisant plusieurs langues romanes? Dans la pratique de l'intercompréhension plurilingue - c'est-à-dire quand chacun comprend les langues des autres et se fait comprendre dans sa (ses) première(s) langue(s) -, nous identifions dans cet article plusieurs types de manifestations interculturelles en référence aux travaux sur la communication exolingue et à la place et au rôle de l'interculturalité dans la perspective actionnelle du Cadre européen commun de référence. Cette catégorisation est ensuite exemplifiée et discutée en prenant appui sur des extraits choisis d'interactions en ligne tirés de la plateforme Galanet.

How can intercultural skills be activated in a collaborative e-learning platform on internet, involving several groups of learners from different countries and different romance languages? In plurilingual cross-comprehension practice - i.e. when everyone understands the other languages and knows how to be understood in his $1^{\text {st }}$ romance language(s) -, we identify in this paper various kinds of intercultural phenomena in reference to exolingual communication and in reference to the place and role of interculturality in the action-oriented approach of the Common European Framework. This categorisation is afterwards exemplified and discussed using some examples of e-interaction gathered from the Galanet platform.

\section{AUTEURS}

\section{CHRISTIAN DEGACHE}

Université Stendhal-Grenoble 3, Lidilem

COVADONGA LÒPEZ ALONSO

Universidad Complutense de Madrid

\section{ARLETTE SÉRÉ}

Universidad Complutense de Madrid 